\title{
Rippled Quasiperpendicular Shock Observed by the Magnetospheric Multiscale Spacecraft
}

\author{
A. Johlander, ${ }^{1,2}$ S. J. Schwartz, ${ }^{3,4}$ A. Vaivads, ${ }^{1}$ Yu. V. Khotyaintsev, ${ }^{1}$ I. Gingell, ${ }^{3}$ I. B. Peng, ${ }^{5}$ S. Markidis, ${ }^{5}$ \\ P.-A. Lindqvist, ${ }^{5}$ R. E. Ergun, ${ }^{4}$ G. T. Marklund, ${ }^{5}$ F. Plaschke, ${ }^{6}$ W. Magnes, ${ }^{6}$ R. J. Strangeway, ${ }^{7}$ C. T. Russell, ${ }^{7}$ H. Wei, ${ }^{7}$ \\ R. B. Torbert, ${ }^{8}$ W. R. Paterson, ${ }^{9}$ D. J. Gershman, ${ }^{9,10}$ J. C. Dorelli, ${ }^{9}$ L. A. Avanov, ${ }^{9}$ B. Lavraud,${ }^{11,12}$ Y. Saito, ${ }^{13}$ \\ B. L. Giles, ${ }^{9}$ C. J. Pollock, ${ }^{9}$ and J. L. Burch ${ }^{14}$ \\ ${ }^{1}$ Swedish Institute of Space Physics, Uppsala 75121, Sweden \\ ${ }^{2}$ Department of Physics and Astronomy, Uppsala University, Uppsala 75120, Sweden \\ ${ }^{3}$ Blackett Laboratory, Imperial College London, London SW7 2AZ, United Kingdom \\ ${ }^{4}$ Laboratory of Atmospheric and Space Physics, University of Colorado, Boulder, Colorado 80303, USA \\ ${ }^{5}$ KTH Royal Institute of Technology, Stockholm 11428, Sweden \\ ${ }^{6}$ Space Research Institute, Austrian Academy of Sciences, Graz 8042, Austria \\ ${ }^{7}$ University of California, Los Angeles, California 90095, USA \\ ${ }^{8}$ University of New Hampshire, Durham, New Hampshire 03824, USA \\ ${ }^{9}$ NASA Goddard Space Flight Center, Greenbelt, Maryland 20771, USA \\ ${ }^{10}$ University of Maryland, College Park, Maryland 20742, USA \\ ${ }^{11}$ Institut de Recherche en Astrophysique et Planétologie, Université de Toulouse, Toulouse 31028, France \\ ${ }^{12}$ Centre National de la Recherche Scientifique, UMR 5277, Toulouse 31400, France \\ ${ }^{13}$ Institute of Space and Astronautical Science, JAXA, Sagamihara 2525210, Japan \\ ${ }^{14}$ Southwest Research Institute, San Antonio, Texas 78238, USA \\ (Received 10 June 2016; published 12 October 2016)
}

\begin{abstract}
Collisionless shock nonstationarity arising from microscale physics influences shock structure and particle acceleration mechanisms. Nonstationarity has been difficult to quantify due to the small spatial and temporal scales. We use the closely spaced (subgyroscale), high-time-resolution measurements from one rapid crossing of Earth's quasiperpendicular bow shock by the Magnetospheric Multiscale (MMS) spacecraft to compare competing nonstationarity processes. Using MMS's high-cadence kinetic plasma measurements, we show that the shock exhibits nonstationarity in the form of ripples.
\end{abstract}

DOI: 10.1103/PhysRevLett.117.165101

Collisionless shocks are abundant in astrophysical plasmas such as around supernova remnants and in our solar system as planetary bow shocks and interplanetary shocks. Shocks thermalize supersonic flows and are effective particle accelerators. Shock physics is influenced by the angle $\theta_{B n}$ between the upstream magnetic field and the shock normal. A quasiperpendicular shock has $\theta_{B n}>45^{\circ}$ and quasiparallel $\theta_{B n}<45^{\circ}$. Above the first critical Mach number, $M_{A} \sim 3$ or less [1], quasiperpendicular shocks reflect a fraction of incident ions which gyrate and return to the shock with sufficient energy to pass downstream, where they are responsible for the bulk of the ion heating.

Under varying upstream conditions, relatively constant shock profiles can move up- or downstream [2]. In contrast, under stable upstream conditions, the motion and structure of a nonstationary shock is nonuniform and changes with time. Nonstationarity, and its role in particle heating and

Published by the American Physical Society under the terms of the Creative Commons Attribution 3.0 License. Further distribution of this work must maintain attribution to the author(s) and the published article's title, journal citation, and DOI. acceleration, is a long-standing issue in shock physics. It was theorized by Auer, Hurwitz, and Kilb [3] and demonstrated in laboratory plasmas [4]. Hybrid simulations by Leroy et al. [5] showed that the shock can become unstable for low ion beta and high Mach number $M_{A}>8$, when the shock over- or underreflects ions, which leads to a new shock forming upstream of an existing shock. Krasnoselskikh et al. [6] examined the critical whistler Mach number above which nonlinear whistler waves cannot exist in the shock ramp. This leads to an intrinsically unsteady shock behavior. In a study using Cluster data, Lobzin et al. [7] demonstrated shock nonstationarity and associated variability in ion reflection. Shock nonstationarity has also been observed at the bow shocks of Mercury [8] and Saturn [9].

Shock nonstationarity can take several forms linked to the underlying microphysical processes. One important kind of nonstationarity is shock rippling. Simulations by Lowe and Burgess [10] showed that the surface of a quasiperpendicular shock supports ripples that propagate along the shock front. Such ripples are potential sites of electron acceleration [11] and influence the ion dynamics [12,13]. Moullard et al. [14] presented evidence of such ripples by exploiting a slow, 
partial crossing of Earth's bow shock made by the Cluster spacecraft. There is a lack of detailed studies of shock ripples.

Here we investigate shock nonstationarity using data from the Magnetospheric Multiscale (MMS) mission [15], which for the first time allows detailed kinetic scale observations of the shock nonstationarity.

Observations.-We study one quasiperpendicular bow shock crossing on October 7, 2015, 11:44 UT. The passage of an interplanetary coronal mass ejection (ICME) compressed the magnetosphere and gave rise to MMS exiting from the magnetosheath into the undisturbed solar wind.

For our analysis, electric field data are provided from the electric field instrument $[16,17]$ and magnetic field data from the fluxgate magnetometer [18], both in the FIELDS instrument suite [19]. Ion data are provided by FPI-DIS (Fast Plasma Investigation-Dual Ion Spectrometer) [20]. Since FPI-DIS is not designed to monitor the solar wind, we use OMNI data for upstream plasma moments.

Figure 1 shows magnetic field and ion data from MMS3. In a few seconds, the plasma transitions from the solar wind to the magnetosheath and the magnetic field magnitude $B$ has a sharp increase, which is typical for a quasiperpendicular shock. The solar wind at this event is characterized by the dense ICME plasma, $n_{u}=29 \mathrm{~cm}^{-3}$ with bulk speed $V_{u}=425 \mathrm{~km} / \mathrm{s}$, and a strong upstream magnetic field $B_{u}=16 \mathrm{nT}$ (see Table I). This inbound shock crossing at 11:44 UT has $\theta_{B n}=83^{\circ}$ and Alfvén Mach number $M_{A}=6.2$. Downstream of the shock, we observe fluctuations in the magnetic field. These fluctuations are correlated with structures in the ion distribution functions.

The shock normal $\hat{\mathbf{n}}$ is determined by the mixed data method $[21,22]$ that uses both the magnetic field and ion velocity upstream and downstream of the shock. This method is better suited than others for nearly perpendicular shocks. In addition, four-spacecraft timing of the shock

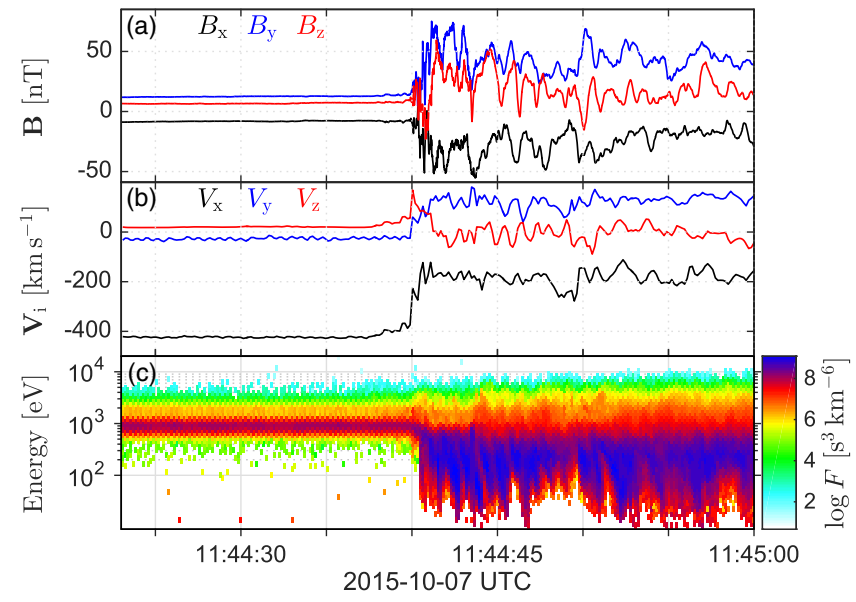

FIG. 1. Overview of the event by MMS3. (a) Magnetic field in geocentric solar ecliptic (GSE) coordinates. (b) Ion bulk velocity in GSE. (c) Ion phase-space density as a function of energy. surface [22] turns out to be impossible due to the fact that the four spacecraft observe rather different shock profiles despite close spacecraft separation. This is an early indication that the shock motion and/or evolution is nonuniform. The resulting normal is within $6^{\circ}$ of the model bow shock normal $[22,23]$. Solar wind and shock parameters including normal vector are presented in Table I.

We use a coordinate system $\hat{\mathbf{n}}, \hat{\mathbf{t}}_{1}, \hat{\mathbf{t}}_{2}$, where $\hat{\mathbf{t}}_{2}=$ $\hat{\mathbf{n}} \times \mathbf{B}_{u} / B_{u}$ and $\hat{\mathbf{t}}_{1}=\hat{\mathbf{t}}_{2} \times \hat{\mathbf{n}} . \mathbf{B}_{u}$ is approximately along $\hat{\mathbf{t}}_{1}$ in the $\hat{\mathbf{n}}-\hat{\mathbf{t}}_{1}$ plane. The upstream convection electric field $\mathbf{E}_{u}$ is approximately along $\hat{\mathbf{t}}_{2}$.

Specular ion reflection.-Earlier studies have shown that, above the first critical fast Mach number, reflected ions are the result of specular reflection off the steep shock ramp [24,25]. The reflected ions gyrate and are accelerated in the shock normal incidence reference frame (NIF) by $\mathbf{E}_{u}$. With MMS for the first time we can analyze the interplay between the reflection process and shock nonstationarity at the relevant temporal and spatial scales even at rapid shock crossings.

Figure 2 shows three snapshots of the ion distribution at different times as a function of $v_{n}, v_{t 1}$, and $v_{t 2}$. The dashed circles in Figs. 2(e)-2(g) show velocities corresponding to $\left|\mathbf{v}-\mathbf{V}_{u}\right|=2\left|\mathbf{V}_{u} \cdot \hat{\mathbf{n}}\right|$, which assumes constant energy in the solar wind frame and specular reflection.

The first snapshot (I), in Figs. 2(b) and 2(e), is from the time when the spacecraft are furthest upstream of the shock but where reflected ions are still observed. As first reported by Paschmann et al. [25], such ions are at the upstream turnaround distance from the shock and are moving purely tangentially to the shock. Figures 2(b) and 2(e) show that the MMS observations are consistent with this prediction. The second snapshot (II) [Figs. 2(c) and 2(f)] is deeper into the shock foot. Here the spread of the reflected ions in normal velocity is larger than in I; this is the unresolved set of outward and returning ions expected within the shock foot. The third snapshot (III) [Figs. 2(d) and 2(g)] is from

TABLE I. Shock and plasma parameters. Values for the upstream plasma parameters are from the OMNI database. A factor $\cos \theta_{V n}$ is included in the Mach numbers.

\begin{tabular}{lc}
\hline \hline Parameter & Value \\
\hline Magnetic field magnitude $B$ & $16 \mathrm{nT}$ \\
Solar wind density $n_{u}$ & $29 \mathrm{~cm}^{-3}$ \\
Solar wind speed $\left|\mathbf{V}_{u}\right|$ & $425 \mathrm{~km} \mathrm{~s}^{-1}$ \\
Alfvén Mach number $M_{A}$ & 6.2 \\
Magnetosonic Mach number $M_{\mathrm{ms}}$ & 4.2 \\
Solar wind ion $\beta_{i, u}$ & 0.48 \\
Shock normal in GSE $\mathbf{n}$ & $(0.880 .46-0.11)$ \\
$\theta_{B n}$ & $83^{\circ}$ \\
$\theta_{V n}$ & $21^{\circ}$ \\
Ion inertial length upstream $d_{i, u}$ & $42 \mathrm{~km}$ \\
Ion gyroperiod upstream $\tau_{c i, u}$ & $4.1 \mathrm{~s}$ \\
Alfvén speed in overshoot, $v_{A, o}$ & $160 \mathrm{~km} \mathrm{~s}^{-1}$ \\
\hline \hline
\end{tabular}




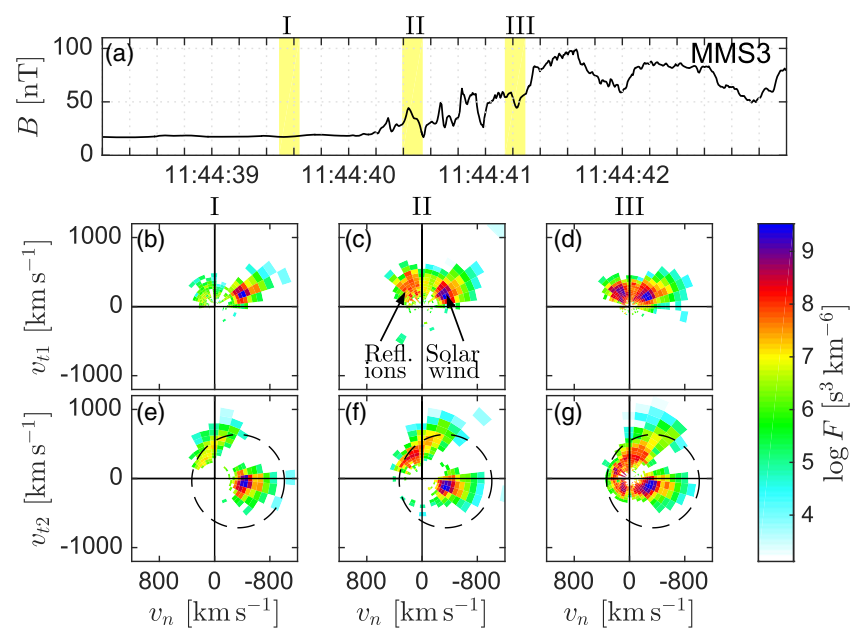

FIG. 2. Snapshots of ion distributions by MMS3. (a) $B$. (b)-(d) Projected ion phase-space density as a function $v_{n}$ and $v_{t 1}$ at times I-III, (e)-(g) as a function $v_{n}$ and $v_{t 1}$. The dashed circles indicate which parts of velocity space reflected ions should occupy, assuming specular reflection.

the shock ramp. Here, solar wind ions are in the process of being reflected. Therefore, we see both almost specular reflected ions with large $v_{n}>0$ and ions returning to the shock with $v_{n}<0$ and large $v_{t 2}>0$. The energy of the returning ions are 3-4 times the energy of incoming solar wind ions.

Thus, we easily reproduce the well-established scenario in which specularly reflected ions are observed from upstream of the foot of the shock to the shock ramp. These reflected ions are accelerated in the direction of $\mathbf{E}_{u}$ and maintain constant speed in the solar wind frame until they penetrate close to the shock ramp.

Shock nonstationarity.-The spacecraft separation is $\sim 25 \mathrm{~km}$, which is approximately $1 / 10$ of the gyroradius of the reflected solar wind ions. Figures 3(a) and 3(b) show the relative positions of the four spacecraft, which are pairwise separated along the shock normal. MMS1 and MMS4 are positioned more upstream than MMS2 and MMS3. MMS2 and MMS3 are predominantly separated along $\hat{\mathbf{t}}_{1}$, i.e., along the magnetic field and tangential to the shock surface.

Figure 3(c) shows $B$ for the four spacecraft. We observe the undisturbed solar wind and later the start of the shock foot at the same time for all spacecraft. However, the shock ramp is observed with a time difference of $\sim 1$ s between the spacecraft pairs. Toward the end of the interval, we see magnetosheath fluctuations at roughly in phase for all spacecraft. As we shall show below, the differences in the vicinity of the shock ramp are due to apparent motion of the shock ramp.

Figure 3(d) shows the normal electric field $E_{n}$, which has been low-pass filtered to more easily see the general structure. $E_{n}$ is strongest at the shock ramp, where ions are reflected, and reaches at most $\sim 30 \mathrm{mV} / \mathrm{m}$ (cf. [26]).
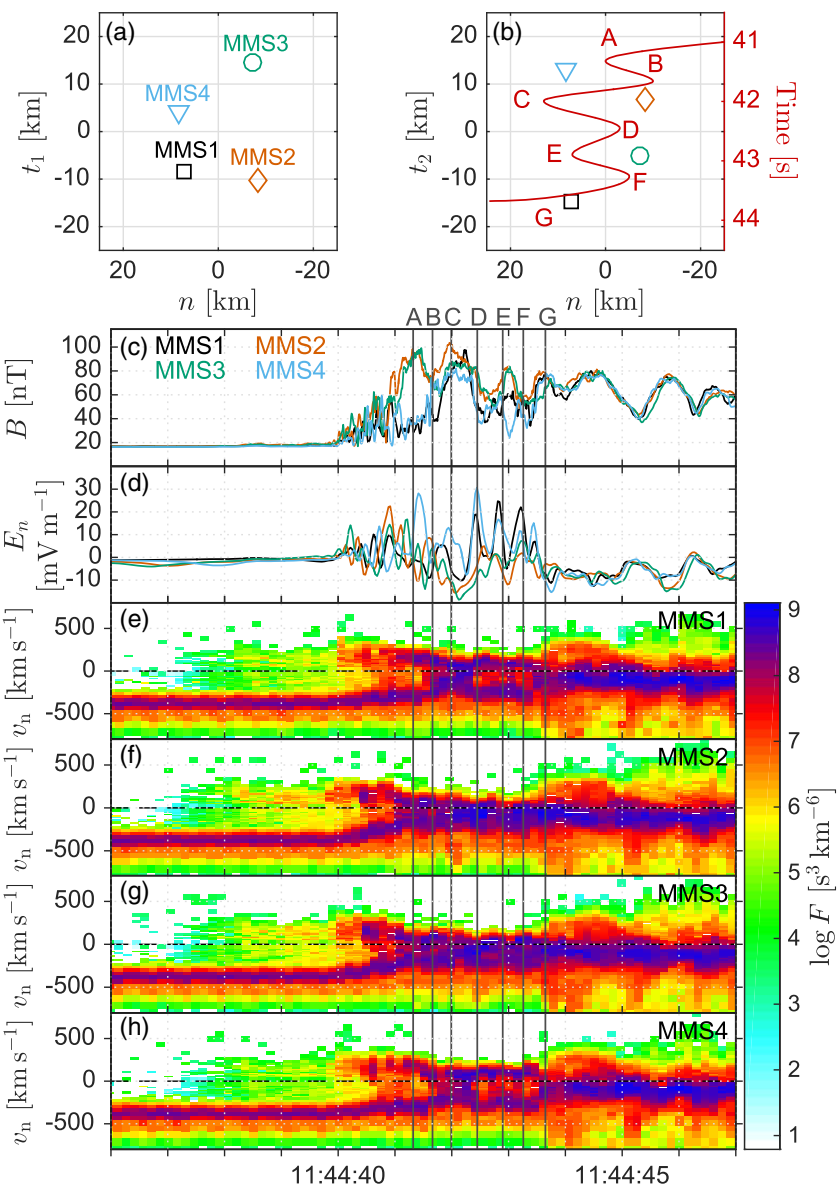

FIG. 3. Four-spacecraft observations of electric, magnetic field, and ions. (a),(b) Spacecraft positions. (b) The red line shows the shock position along $\hat{\mathbf{n}}$ over time. (c) $B$. (d) $E_{n}$. (e)-(h) Ion phasespace density as functions of the normal speed averaged over tangential velocities.

Figures 3(e)-3(h) show the ion phase-space density as a function of normal speed. Reflected ions are first observed by all four spacecraft at the same time, $\sim 3 \mathrm{~s}$ before the shock ramp. The number of reflected ions is not steady, including both smooth variations over $\sim 1 \mathrm{~s}$ in the initial magnetically quiet period and a significant increase coincident with the beginning of the magnetic shock foot.

At the shock ramp, ion reflection is observed where ions go from negative normal speed to positive. After their encounter with the overshoot at time $C$, MMS1 and MMS4 observe partial ion phase-space holes near $v_{n}=0$ between times $D$ and $G$. These ion distributions are similar to the interval at and immediately upstream of the shock ramp (times $A$ and $B$ ). MMS1 and MMS4 also observe peaks in $E_{n}$ like at the first shock ramp. We conclude that MMS1 and MMS4 have returned to upstream of the shock rather than passing through the overshoot into the downstream region.

The combination of high-resolution field and ion observations during this brief ( $5 \mathrm{~s}$ in total) shock encounter 
enables us to reconstruct the apparent shock motion, the red line in Fig. 3(b). While this looks like the result of a simple planar out-in motion of the shock, we show below that the shock front is not planar.

Ripples. - We have shown that two spacecraft go from the solar wind to the shock overshoot and then back upstream. We will now show that this motion is due to ripples propagating along the shock surface rather than larger scale motion of a planar shock front.

The apparent shock motion is indicated in Fig. 4, where OUT means sunward and IN means anti-sunward. We determine this motion from the oscillations in the normal magnetic field $B_{n}$, observed by MMS2 and MMS3. These oscillations can be matched to the apparent in and out motion of the shock seen in the ion phase-space signatures in Fig. 3. Changes in $B_{n}$ can be attributed to a change in the local normal vector of the shock. MMS2 and MMS3 both observe dips in $B_{n}$ during outward motions and peaks during inward motion. $B$ increases as the spacecraft move toward the peak of the overshoot during outward shock motion and decreases during inward shock motion. The oscillations in the magnetic field are consistent with surface waves or ripples moving along the shock surface and which decrease in amplitude away from the shock overshoot [10], so they are not simultaneously seen as clearly by MMS1 and MMS4.

We determine the velocity of the ripples by timing the variations in $B_{n}$ and $B$ between MMS2 and MMS3 and

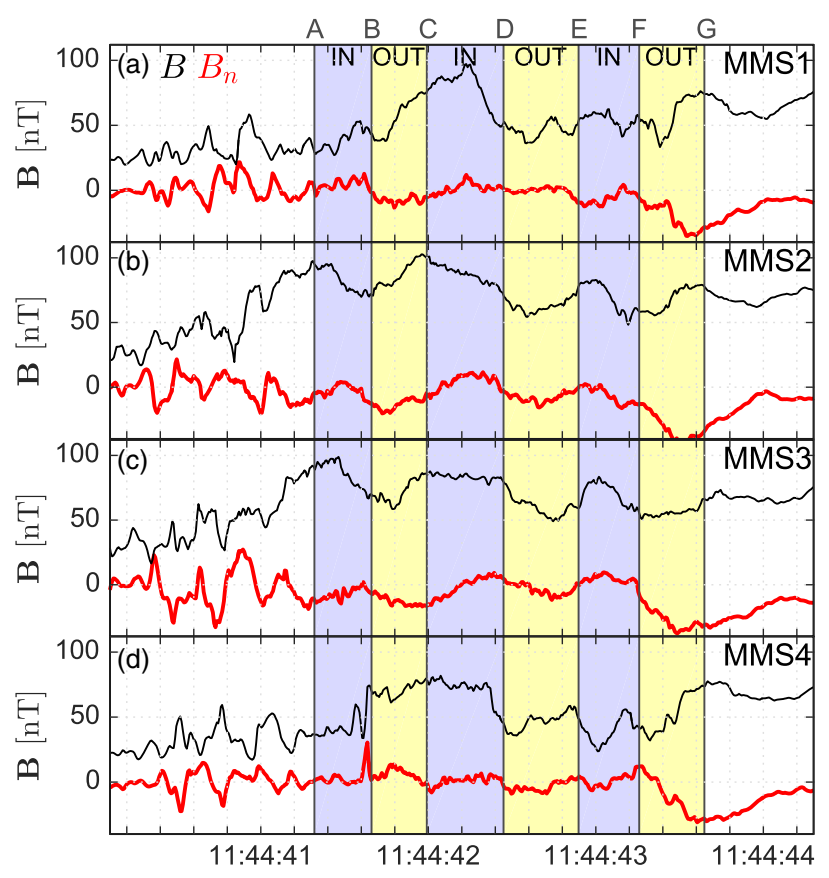

FIG. 4. $\quad B$ (black line) and $B_{n}$ (red line) for all four spacecraft during the shock crossing. MMS2 and MMS3 stay in the overshoot for more than $2 \mathrm{~s}$, and both observe oscillations in $B_{n}$ due to the rippling of the shock surface. The apparent shock motion upstream is indicated as OUT and downstream as IN. assuming that the ripples propagate along $\hat{\mathbf{t}}_{1}$, i.e., along $\mathbf{B}_{u}$ [10]. The ripples are propagating parallel, rather than antiparallel, to $\mathbf{B}_{u}$ with a phase speed in the NIF $v_{r}=105 \mathrm{~km} \mathrm{~s}^{-1}$. This is slightly lower than the simulation result $v_{r}=v_{A, o} \sim 160 \mathrm{~km} \mathrm{~s}^{-1}$ [10].

The average period of the ripples in the NIF is $T_{r}=1.7 \mathrm{~s}$. This corresponds to $T_{r}=0.4 \tau_{c i, u}$, which is close to $\sim 0.3 \tau_{c i, u}$ predicted by Lowe and Burgess [10]. Knowing $T_{r}$ and $v_{r}$, we find the ripple wavelength $\lambda_{r}=175 \mathrm{~km}$, or $4.2 d_{i, u}$, in good agreement with the range (4-8) $d_{i, u}$ reported by Lowe and Burgess [10].

The approximate spatial peak-to-peak amplitude $A$ of the ripples is implicitly derived from Fig. 3(b) to be 10-20 km. This corresponds to $A=(0.25-0.5) d_{i, u}$, which is close to $(0.5-1) d_{i, u}$ predicted by Ofman and Gedalin [27]. We also estimate $A$ from the size of the $B_{n}$ oscillations in Figs. 4(b) and 4(c). Assuming sine-like ripples,

$$
A=\frac{\lambda \Delta B_{n} / B_{0}}{\pi \sqrt{4-\left(\Delta B_{n} / B_{0}\right)^{2}}}
$$

where $\Delta B_{n}$ is the peak-to-peak variations in $B_{n}$ and $B_{0}$ is the magnetic field magnitude in the $\hat{\mathbf{n}}-\hat{\mathbf{t}}_{1}$ plane. With $\Delta B_{n}=20 \mathrm{nT}$ and $B_{0}=70 \mathrm{nT}, A=8 \mathrm{~km}$, which is in good agreement with the previous estimate. Figure 5 summarizes the quantities derived above and shows the spacecraft trajectories through the rippled shock. The overall impression of unidirectional passage through the shock foot, ramp, overshoot, and undershoot into the downstream region is incorrect; instead, the spacecraft cross the shock several times due to the ripples.

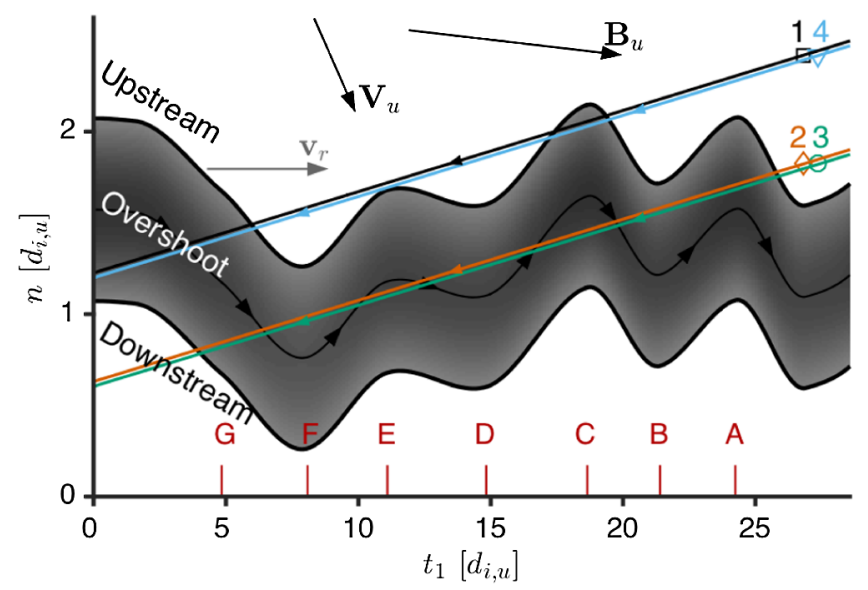

FIG. 5. Sketch of the shock crossing in the $\hat{\mathbf{n}}-\hat{\mathbf{t}}_{1}$ plane. The overshoot of the shock is illustrated by a rippled ribbon with a magnetic field, and velocities in the spacecraft frame are shown by arrows and the magnetic field magnitude by shading. The spacecraft trajectories in the ripple frame are illustrated by colored lines. The tangential spacecraft positions $t_{1}$ at times $A-G$ are marked in the bottom. 
At time $G$ in Figs. 3 and 4, all four spacecraft cross the overshoot for the last time. This time, the spacecraft cross at the downstream part of a ripple. $B$ in this part of the overshoot is $\sim 20 \%$, or $\sim 1 B_{u}$ lower than the overshoot first encountered, as reported by Ofman and Gedalin [28]. After the spacecraft have crossed the overshoot at time $G$, the plasma is more thermalized. Here, the magnetic field profiles for all spacecraft become similar and show compressional structures. High-energy reflected ions with $v_{n}<$ $500 \mathrm{~km} / \mathrm{s}$ are in phase with these structures. We interpret these structures as part of the ripples and the reflected ions as an effect of the shock surface moving relative to the spacecraft.

Conclusions. - We use the high-resolution data from the four closely spaced MMS spacecraft to investigate the structure of a supercritical, quasiperpendicular shock. The observed variations in magnetic, electric field, and ion distributions can be explained by ripples moving along the shock surface. Quantitative analysis reveals that, unlike earlier reports [14], these ripples match the kinetic scale and dispersion properties of ripples seen in $2 \mathrm{D}$ hybrid simulations [10]. Our observations, for the first time, provide a detailed picture of a rippled, quasiperpendicular shock at kinetic scales.

We show that the observed reflected ions upstream of the shock are specularly reflected and accelerated in the solar wind electric field, consistent with previous studies. In addition, we show that the simultaneous presence of incoming and reflected ion beams is a good indicator that spacecraft is upstream of the shock overshoot.

Future work should investigate the role of waves and full 3D electric fields in mediating the shock energy partition through particle heating and dynamics.

For MMS data, see [29].

The OMNI data were obtained from the GSFC/SPDF OMNIWeb interface [30].

We thank the entire MMS team and instrument PIs for data access and support. The IRAP contribution to MMS was funded by CNES and CNRS. S. J. S. gratefully acknowledges the receipt of a Leverhulme Trust Research Fellowship. This study was supported by Swedish National Space Board Contracts No. 139/12 and No. 97/13.

[1] J. P. Edmiston and C. F. Kennel, J. Plasma Phys. 32, 429 (1984).

[2] M. Maksimovic, S. D. Bale, T. S. Horbury, and M. André, Geophys. Res. Lett. 30, 1393 (2003).
[3] P. L. Auer, H. Hurwitz, Jr., and R. W. Kilb, Phys. Fluids 5, 298 (1962).

[4] D. L. Morse, W. W. Destler, and P. L. Auer, Phys. Rev. Lett. 28, 13 (1972).

[5] M. M. Leroy, D. Winske, C. C. Goodrich, C. S. Wu, and K. Papadopoulos, J. Geophys. Res. 87, 5081 (1982).

[6] V. V. Krasnoselskikh, B. Lembège, P. Savoini, and V. V. Lobzin, Phys. Plasmas 9, 1192 (2002).

[7] V. V. Lobzin, V. V. Krasnoselskikh, J.-M. Bosqued, J.-L. Pinçon, S. J. Schwartz, and M. Dunlop, Geophys. Res. Lett. 34, L05107 (2007).

[8] T. Sundberg, S. A. Boardsen, J. A. Slavin, V. M. Uritsky, B. J. Anderson, H. Korth, D. J. Gershman, J. M. Raines, T. H. Zurbuchen, and S. C. Solomon, J. Geophys. Res. 118, 6457 (2013).

[9] A. H. Sulaiman, A. Masters, M. K. Dougherty, D. Burgess, M. Fujimoto, and G. B. Hospodarsky, Phys. Rev. Lett. 115, 125001 (2015).

[10] R.E. Lowe and D. Burgess, Ann. Geophys. 21, 671 (2003).

[11] T. Umeda, M. Yamao, and R. Yamazaki, Astrophys. J. 695, 574 (2009).

[12] Z. W. Yang, B. Lembège, and Q. M. Lu, J. Geophys. Res. 117, A07222 (2012).

[13] Y. Hao, Q. Lu, X. Gao, and S. Wang, Astrophys. J. 823, 7 (2016).

[14] O. Moullard, D. Burgess, T. S. Horbury, and E. A. Lucek, J. Geophys. Res. 111, A09113 (2006).

[15] J. L. Burch, T. E. Moore, R. B. Torbert, and B. L. Giles, Space Sci. Rev. 199, 5 (2016).

[16] P.-A. Lindqvist et al., Space Sci. Rev. 199, 137 (2016).

[17] R. E. Ergun et al., Space Sci. Rev. 199, 167 (2016).

[18] C. T. Russell et al., Space Sci. Rev. 199, 189 (2016).

[19] R. B. Torbert et al., Space Sci. Rev. 199, 105 (2016).

[20] C. Pollock et al., Space Sci. Rev. 199, 331 (2016).

[21] B. Abraham-Shrauner, J. Geophys. Res. 77, 736 (1972).

[22] S. J. Schwartz, ISSI Sci. Rep. Ser. 1, 249 (1998).

[23] M. H. Farris, S. M. Petrinec, and C. T. Russell, Geophys. Res. Lett. 18, 1821 (1991).

[24] L. C. Woods, J. Plasma Phys. 3, 435 (1969).

[25] G. Paschmann, N. Sckopke, J. R. Asbridge, S. J. Bame, and J. T. Gosling, J. Geophys. Res. 85, 4689 (1980).

[26] S. Walker, H. Alleyne, M. Balikhin, M. André, and T. Horbury, Ann. Geophys. 22, 2291 (2004).

[27] L. Ofman and M. Gedalin, J. Geophys. Res. 118, 5999 (2013).

[28] L. Ofman and M. Gedalin, J. Geophys. Res. 118, 1828 (2013).

[29] https://lasp.colorado.edu/mms/sdc/public/.

[30] http://omniweb.gsfc.nasa.gov. 